\title{
Concepción de un sistema de iluminación inteligente en Smart Cities como Proyecto Fin de Carrera de Ingeniería Informática
}

\author{
Víctor Martín, Miguel Damas, Jesús González, Héctor Pomares \\ ${ }^{1}$ Departamento de Arquitectura y Tecnología de Computadores \\ Granada, España \\ \{vicmr, mdamas, jesusgonzalez, hector \}@ugr.es
}

\begin{abstract}
Resumen. Este trabajo presenta el proyecto fin de carrera "Iluminación inteligente en las ciudades del futuro" defendido en Septiembre de 2013 en la titulación Ingeniería Informática de la Universidad de Granada, un proyecto distintivo y que ofrece al alumno una formación y una madurez añadida. Durante el desarrollo de este proyecto se presenta un sistema de gestión de farolas inteligentes basado en lámparas LED, diseñadas para facilitar su despliegue en las instalaciones existentes. El enfoque propuesto, basado en tecnologías de comunicación inalámbrica, permite reducir al mínimo el coste de la inversión de los sistemas de cable tradicionales. Gracias a la realización de este proyecto, se consigue aprender tecnologías y sistemas que no son tratados en la carrera, permitiendo al alumnado conocer uno de los temas más demandados en la actualidad.
\end{abstract}

Palabras Clave: Ciudades inteligentes, Alumbrado Inteligente, Ingeniería Informática.

\begin{abstract}
This paper presents the final project "Intelligent lighting in the cities of the future" presented in September 2013 at the Computer Engineering degree at the University of Granada, a distinctive project that offers students an added training and maturity. During the development of this project a management system based on smart streetlights LED lamps designed for easy deployment in existing facilities is presented. The proposed approach, based on wireless communication technologies, can minimize the investment cost of traditional cable systems. Thanks to this project, one gets to learn technologies and systems that are not treated in the career, allowing the students to know one of the most popular topics today.
\end{abstract}

Keywords: Smart City, Smart Lighting, Computer Engineering. 


\section{Introducción}

En las antiguas titulaciones de Ingeniería Informática existía una carencia en cuanto a que los temas de actualidad no se estudiaban, aunque con la implantación del nuevo Grado se han propuesto asignaturas que estaban más relacionadas, como puede ser la asignatura "Sistemas Empotrados". La mayoría de las veces, los estudiantes eligen proyectos fin de carrera relacionados con temas conocidos y anteriormente tratados que realmente no aportan nada a la formación del alumno. Se debería de proponer la realización de proyectos fin de carrera orientados a temas de actualidad y que, en cierto modo, no se hayan tratado específicamente en la carrera, con el fin de dotar al estudiante de una competencia extra y acercarlo un poco más a los temas que se va a encontrar en su futuro profesional. Sin ir más lejos, este proyecto fin de carrera ha supuesto un gran aporte académico, ya que se ha tratado con tecnologías novedosas y hardware (tema bastante olvidado en las antiguas titulaciones de Ingeniería Informática). Por este motivo, a continuación el primer autor de este trabajo presenta un proyecto fin de carrera que esta fuera de lo normalmente realizado.

La urbanización ha aumentado encarecidamente en los últimos años, y las previsiones muestran que la migración de la población hacia las zonas urbanas no va a decrecer [1]. Encontrar ciudades con decenas de millones de habitantes ya no es algo extraño. Esta concentración de población en las ciudades plantea numerosos desafíos en términos de gobernabilidad de la ciudad y la vida de las personas. Como consecuencia, soluciones "más inteligentes" son necesarias para mejorar, día a día, las ciudades. Las ciudades inteligentes o Smart Cities han reclamado un lugar central en las agendas de innovación de los gobiernos, organizaciones de investigación y proveedores de tecnología, lo que plantea desafíos únicos y difíciles. Desde la perspectiva de la investigación, las ciudades inteligentes son inherentemente interdisciplinares: requieren la investigación y la cooperación de varias disciplinas, que abarcan desde la economía a las ciencias sociales, y desde la política a la gestión de infraestructuras. Por tanto, el paradigma de Smart City se puede considerar como un sistema distribuido en el que las diferentes fuentes de información proporcionan datos a un conjunto de aplicaciones que los utilizan para la elaboración de respuestas a nivel estratégico y táctico.

El alumbrado público en España representa el 10\% del consumo total de energía en la iluminación y se sitúa en 116 kilovatios por año y habitante [2], en comparación con los $91 \mathrm{~kW}$ o $43 \mathrm{~kW}$ en Francia y Alemania, respectivamente [3]. Las últimas cifras disponibles del Ministerio de Industria, Turismo y Comercio suponen un consumo de energía eléctrica de $3.630 \mathrm{GWh}$ / año para el conjunto de España. Vale la pena señalar que, en total, había 4.8 millones de puntos de luz estimados en el año 2010, y que un tercio del alumbrado público se basa en tecnologías anticuadas e ineficientes. La iluminación pública tiene el mayor impacto en el consumo de energía de un municipio, y puede representar hasta el 54\% del consumo total de energía y el $61 \%$ del consumo eléctrico de las instalaciones municipales. La importancia de las instalaciones de alumbrado público es tal que, en algunos municipios, este hecho ahora representa hasta el $80 \%$ de la electricidad consumida y hasta el $60 \%$ del presupuesto de consumo de energía del municipio. 
Luego, teniendo en cuenta las anteriores consideraciones, la innovación en los sistemas de gestión eficientes de alumbrado público es una necesidad, de ahí que en este trabajo se proponga una solución escalable, integral y eficiente que proporcione la iluminación sólo cuando sea necesaria (según las condiciones meteorológicas instantáneas o la presencia de personas y vehículos), con el objetivo de reducir los costes relacionados en los municipios, y así ayudar a la recuperación económica. Además, el nivel de iluminación será convenientemente regulado, evitando de esta manera el deslumbramiento, y el coste de la instalación será reducido al mínimo utilizando las comunicaciones inalámbricas. Todas estas mejoras tienen que ser consideradas a fin de lograr una reducción significativa del consumo de energía en la iluminación y por lo tanto contribuir al desarrollo sostenible. Además, es un sistema autónomo, donde se presenta un enfoque integral que garantiza la estandarización, la interoperabilidad y la adaptación a las necesidades y escenarios. Este es un aspecto no funcional pero distintivo respecto a otras soluciones antes mencionadas, que están diseñadas específicamente para un escenario concreto, y con pocas posibilidades de migración a otro entorno.

El resto del articulo está organizado de la siguiente forma: en la sección 2 se introduce el concepto de Smart City y se describe el servicio en el que está basado este artículo; en la sección 3 se analiza el diseño adoptado para el desarrollo de una plataforma de alumbrado inteligente; en la sección 4 se presenta la implementación realizada para dicho sistema y en la sección 5 se muestran los resultados obtenidos. Finalmente se termina el artículo presentando las conclusiones generales y la bibliografía utilizada.

\section{Smart City}

En las antiguas titulaciones de Ingeniería Informática el concepto de Smart City pasaba totalmente inadvertido pero en realidad es uno de los temas de mayor impacto en la actualidad, por lo que tener conocimiento de este nuevo paradigma proporciona un aporte extra a la formación del alumno. A continuación, se presenta dicho concepto.

Más de la mitad de la población del planeta vive en ciudades. Se espera que la cifra supere el sesenta por ciento en 2050. La calidad de vida de miles de millones de personas dependerá de su capacidad para innovar, y ofrecer mejores servicios y oportunidades a sus habitantes (por ejemplo, ciudades más limpias, más cómodas, más verdes, y en definitiva más inteligentes).

El modelo actual de ciudad está en crisis. En general son complejas, contaminantes y caras. Las nuevas tecnologías ofrecen la oportunidad de revolucionar su gestión y sus procesos y con ellos mejorar la vida de sus ciudadanos, e incluso la salud del planeta. Las ciudades consumen tres cuartas partes de la energía que se produce en el mundo y son responsables del $80 \%$ del dióxido de carbono que se emite a la atmósfera. Mejorar la eficiencia de las urbes es, por lo tanto, pieza clave en la lucha contra el cambio 
climático. Hacerlo exige un esfuerzo multidisciplinar, que conecte todos sus elementos (personas, edificios, vehículos e infraestructuras), y la tecnología para hacerlo realidad ya existe. A continuación, se muestran una serie de ejemplos donde se adopta el concepto de Smart City [4].

\section{- Exprimir la energía}

La mayor parte de la energía que se produce y no se consume al momento se pierde. Una red eléctrica inteligente (Smart Grid) puede ayudar a gestionar su distribución para que llegue a los lugares en los que hace falta solo cuando ésta se necesite. La mejor manera de aprovechar la energía, especialmente cuando proviene de fuentes renovables, es producirla cuando las condiciones son óptimas, almacenarla y después consumirla cuando y donde sea necesario.

\section{- Energía móvil}

Las baterías de los coches, especialmente los eléctricos, permiten acumular el excedente de energía que se produce, e introducirla en el sistema en el momento en que se necesita. Una tecnología así permitiría exprimir al máximo las fuentes renovables, y reducir el trabajo de las centrales de combustibles fósiles. La mayoría de las marcas de automóviles ya disponen de vehículos con esta capacidad.

\section{- Aparcamientos en red}

Plazas de aparcamiento que pueden saber cuándo están libres, y actualizar su estado para que cualquiera pueda encontrarlas gracias a su Smartphone es otro de los servicios que ofrecen las Smart Cities. Por ejemplo, la empresa catalana Urbiotica ya ha instalado un sistema así en Niza (Francia).

\section{- Jardines que se riegan solos}

El riego de parques y jardines se puede automatizar con la instalación de sensores de humedad y temperatura, para que solo rieguen cuando sea necesario. Los mismos equipos pueden, además, avisar de posibles enfermedades en las plantas, y cómo resolverlas. Gracias a este servicio, las ciudades maximizan el ahorro de agua y el mantenimiento de la misma.

\section{- Recogida inteligente de residuos}

Otro servicio interesante que pueden ofrecer las Smart Cities es la recogida inteligente de residuos. Por ejemplo, la empresa Urbiotica ha instalado en Sant Cugat del Vallés un sistema que diseña rutas para que los camiones de la basura sólo recojan los contenedores que están llenos o casi llenos. Así consiguen que se ahorre en combustible y viajes, además de reducir la contaminación acústica de la ciudad. 


\section{- Patrullas dinámicas de la policía}

Gracias a las Smart Cities, ciudades como Memphis (EE.UU.) ha reducido un 30 por ciento sus tasas de delincuencia grave gracias a un sistema de IBM que geo localiza los crímenes que se cometen en la ciudad, y diseña las rutas más eficientes para los cuerpos de seguridad.

\section{- Sistema de alumbrado inteligente}

Por último, se va a presentar el sistema de alumbrado inteligente en la Smart City [5], servicio en el que se centra este artículo. La idea es conseguir un ahorro energético optimizando el uso que se le da al alumbrado público. Para ello se necesita disponer de sensores que detecten la presencia de personas y que midan la cantidad de luminosidad, para así poder regular la intensidad de luz que emiten las farolas, es decir, conseguir de forma automática la cantidad de luz artificial justa, en función de la presencia y la cantidad de luz natural que tenemos en cada momento. En este sentido, la iluminación LED ofrece un mayor rendimiento, más horas de vida útil, consumo menor de energía y son menos contaminantes que las actuales farolas de vapor de sodio y vapor de mercurio usadas para iluminar.

\section{Diseño de un sistema para el alumbrado inteligente}

De nuevo y desde el punto de vista de un estudiante recién titulado, a pesar de la importancia de los elementos que se van a presentar a continuación, en gran parte de las titulaciones de Ingeniería Informática existía una carencia importante. Realmente, para un alumno es difícil aprender elementos de tan bajo nivel por sí mismo, pero por el contrario, una formación autodidacta proporciona una madurez al estudiante que le permitirá la resolución de problemas cada vez mayores por sus propios medios.

La base sobre la que se sustenta la plataforma de alumbrado inteligente está formada por los siguientes elementos:

- Dispositivo con microcontrolador que nos permite administrar y gestionar nuestro sistema. Se ha utilizado el Freescale de tercera generación MC13224 [6], el cual incorpora un procesador de baja potencia de $2.4 \mathrm{GHz}$ de frecuencia, núcleo ARM7 de 32, aceleración hardware para las aplicaciones IEEE 802.15.4, temporizadores programables e interrupciones externas en tiempo real.

- Sistema Operativo instalado en los dispositivos empotrados, que nos facilita entre otras muchas cosas un protocolo de comunicación indispensable para la realización de esta tarea.

- Sensores que permiten conocer características del mundo exterior y dotar al sistema de inteligencia. 
- Actuadores que permiten reflejar el resultado de la inteligencia del sistema.

El sistema operativo elegido para el desarrollo del sistema de alumbrado inteligente es Contiki, que es un sistema operativo multitarea compacto de código abierto, altamente portable para sistemas empotrados en red y redes de sensores inalámbricas [7]. Necesita de un kernel, librerías, un programa de carga y una serie de procesos para poder funcionar, y ofrece las siguientes características:

- Los programas pueden ser sustituidos mientras el dispositivo está en funcionamiento.

- La comunicación entre procesos siempre se realiza a través del kernel.

- El kernel no proporciona una capa de adaptación con el hardware sino que deja a las aplicaciones comunicarse directamente con el hardware.

Como se ve en la Figura 1 el sistema Contiki está dividido en dos módulos: el núcleo (core) y la aplicación (loaded program). La partición se realiza durante la compilación y es específica del mapa de memoria de cada plataforma. Normalmente, el núcleo está formado por el kernel, el cargador de la aplicación (program loader), el entorno de ejecución del lenguaje de programación de la aplicación, y una pila de comunicación con drivers para el hardware de comunicación.

ROM

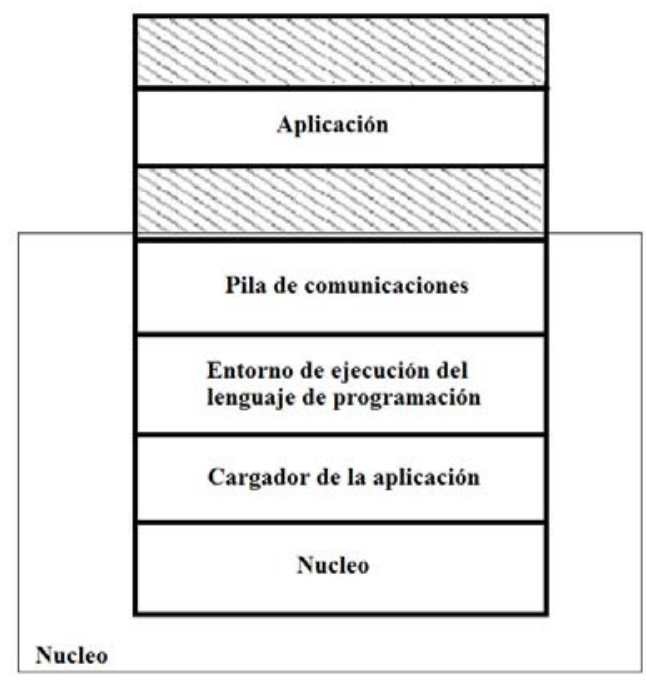

Figura 1. Partición entre el núcleo y programas cargados.

El kernel de Contiki consiste en un sistema de planificación de eventos de bajo consumo que reparte eventos entre los procesos en ejecución, y que periódicamente llama a una serie de procesos que controlan dichos eventos. El kernel soporta dos 
tipos de eventos: asíncronos y síncronos. Los asíncronos son colocados en una cola y se envían a los procesos un poco más tarde. Los eventos síncronos son similares a los asíncronos pero hacen que el proceso objetivo sea inmediatamente programado para ser ejecutado.

Además de lo ya mencionado, el kernel proporciona un sistema polling o de control de eventos. Este puede verse como un sistema de prioridad de eventos que hace que sean programados entre los eventos asíncronos. Suele ser usado por procesos que se encuentran "cerca" del hardware para controlar de forma periódica el estado de los dispositivos de dicho hardware. Por ejemplo, si se usa un puerto serie se tendrá que utilizar dicho mecanismo para ver el flag de recepción del UART, para comprobar si ha llegado un carácter y en caso afirmativo procesarlo.

En resumen, se ha elegido Contiki porque es un Sistema Operativo para redes WSN relativamente ligero. Ofrece soporte para redes IPv6 de forma nativa (6LoWPAN) así como IPv4, CoAP y una pila propia para comunicaciones (entre otras muchas capacidades). El modelo de programación que ofrece es dirigido por eventos y las aplicaciones se programan en lenguaje C. Además, Contiki implementa Protothreads, que permiten la ejecución secuencial de código en sistemas dirigidos por eventos, permitiendo que la ejecución se bloquee y reanude según los eventos dirigidos, evitando la necesidad de implementar complicadas máquinas de estado, como sucede en sistemas operativos similares. También implementa un cargador de módulos dinámicos en tiempo de ejecución, lo que permite modificar el comportamiento de un nodo en tiempo de ejecución.

\subsection{Arquitectura del sistema}

Una vez se ha presentado la idea general del sistema, se está en disposición de presentar la arquitectura llevada a cabo. Se van a identificar y describir los diferentes componentes, poniendo nombres a algunos de ellos para evitar futuras confusiones y una mejor identificación.

\subsubsection{Subsistema Web}

Hoy en día, uno de los temas más demandados en la vida profesional de un informático son los sistemas web y, por lo tanto, cuando un alumno realiza la carrera de Ingeniería Informática espera recibir una formación adecuada sobre esta temática. En el nuevo plan de estudios del grado, han sido incluidas asignaturas como "Servidores Web de Altas Prestaciones" que permiten al alumno aprender a diseñar, instalar y configurar servidores de altas prestaciones, pero de nuevo, en las antiguas titulaciones de Ingeniería Informática solo se podía aprender haciendo cursos de libre configuración. 
Concretamente, en este proyecto se ha diseñado un sistema que permite al usuario interactuar con cada una de las farolas registradas realizando acciones tales como: visualizar el estado de las farolas, cambiar la intensidad lumínica, cambiar el máximo de luz permitido, etc. Al tratarse de un Subsistema Web, es accesible desde cualquier ordenador o dispositivo móvil, con lo cual tenemos un gran abanico de posibilidades para coordinar nuestro sistema (ver figura 2).

\subsubsection{Dispositivo Microcontrolador}

Se trata de un dispositivo que va a desempeñar dos roles distintos dependiendo de si está conectado únicamente a los sensores y actuadores (en este caso actuará como dispositivo hoja), o va a ser conectado únicamente al pc (en este caso actuará como dispositivo coordinador).

Dispositivo coordinador. Este dispositivo será el encargado de gestionar y dirigir todos los dispositivos hoja. Su función principal es recibir los mensajes del subsistema web, por el puerto serie, y reenviar los mensajes al dispositivo hoja correspondiente mediante el protocolo de comunicación 6lowpan [8].

Dispositivo hoja. Se pretende que cada farola tenga acoplado uno de estos dispositivos para tener un control de lo que está midiendo en cada momento, pudiendo notificar estas medidas a otros dispositivos hoja para tener una total conexión entre ellos, a la vez que notifica al dispositivo coordinador para tener centralizados los datos de todos los sensores y qué este tome las decisiones que estime oportunas conforme a las reglas que tenga configuradas (ver figura 3).

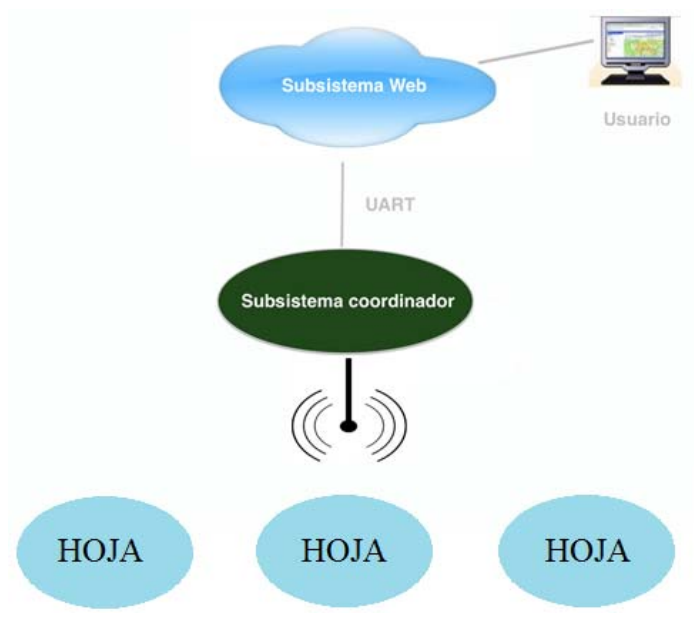

Figura 2. Arquitectura del sistema. 
Este dispositivo está compuesto por un equipo de medida formado por tres componentes distintos. Uno de ellos contiene el sensor de luminosidad (LDR), el otro posee una pantalla de LED que actuará como farola, por último se dispone de un sensor de movimiento (PIR) para detectar los transeúntes. Tanto el sensor de luminosidad (LDR) como el sensor de movimiento (PIR) proporcionarán medidas que permitirán regular la intensidad de la pantalla de LED acorde a las circunstancias.

- Sensor Luminosidad (LDR). Gracias a este sensor se obtendrá la cantidad de luz ambiental cada vez que se necesite. Este sensor ayudara a encender la farola cuando la luz ambiente decaiga por debajo de un cierto umbral.

- Sensor Movimiento. Gracias a este sensor se detectará el movimiento de los transeúntes en las calles. Cada vez que el sensor proporcione un valor positivo indicando que hay movimiento en su zona, la farola se encenderá si la luz ambiental es baja, para que los transeúntes puedan pasear con total normalidad.

- Placa de LEDs. Permite simular el efecto de una farola, tanto el encendido y apagado como la atenuación de la misma.

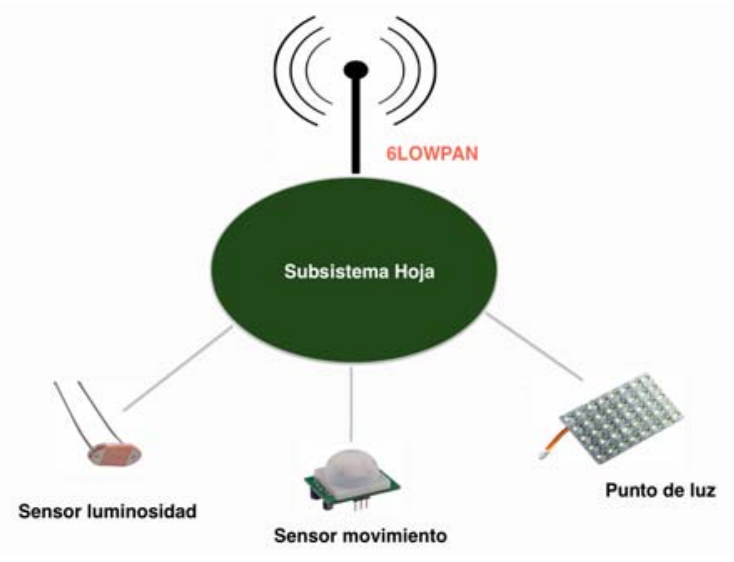

Figura 3. Subsistema hoja.

\section{Implementación del sistema}

A continuación, se muestra la implementación del sistema de control de luz inteligente basado en la arquitectura presentada en la sección anterior. 


\subsection{Subsistema Web}

En primer lugar, el subsistema web está formado por un PC con apache conectado a internet y una mota coordinadora en un puerto serie. Es el encargado de mantener el contacto directo con el usuario. Bajo este subsistema, mostrado en la figura 4, podemos realizar cualquier acción sobre el alumbrado. Este subsistema mostrara las distintas farolas instaladas en una mapa, al pinchar sobre una determinada farola mostrara un menú donde se podrá actualizar la intensidad de esta farola o incluso cambiar el máximo de intensidad que tiene esa farola. Cuando el usuario indique la acción que quiere realizar sobre esta farola, el sistema prepara el mensaje formado por (acción, ip). En primer lugar consultará la IP de la farola seleccionada y en segundo lugar mandará por la UART la dupla (acción, ip) al dispositivo controlador.

Además de estas funcionalidades, el subsistema proporciona al usuario la posibilidad de registrar/eliminar nuevas farolas y de conocer el estado actual de las farolas que tiene registradas.

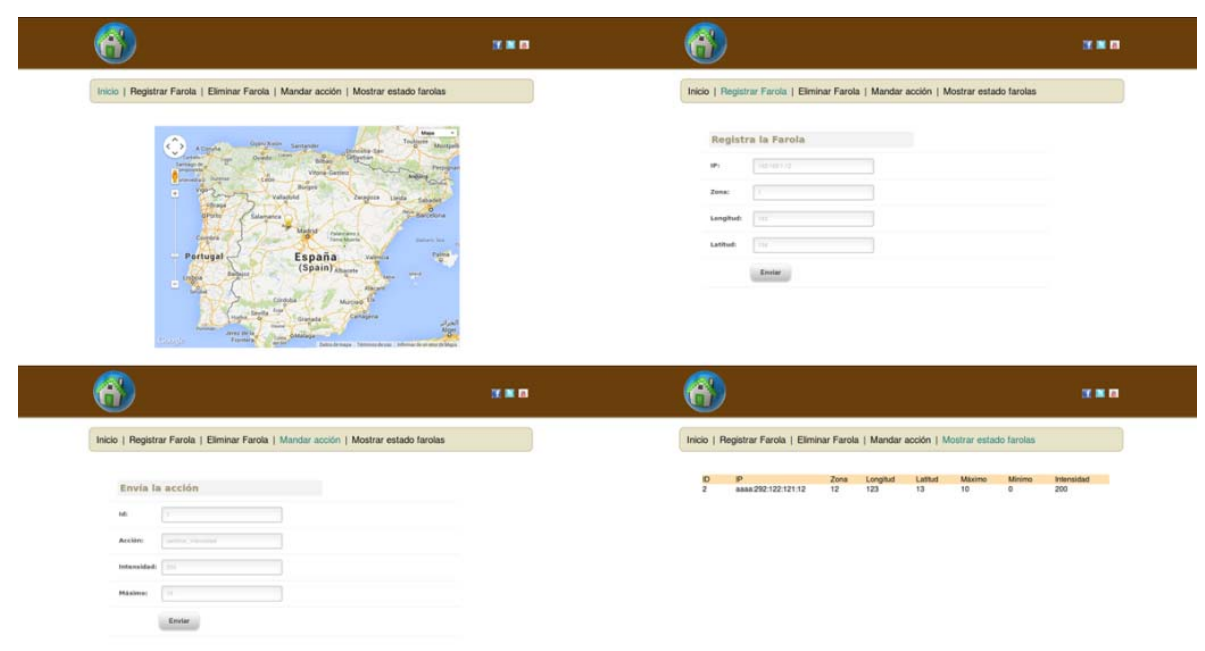

Figura 4. Interfaz gráfica subsistema web.

\subsection{Subsistema Coordinador}

A continuación se describirá el subsistema coordinador, el cual está formado por una mota conectada por el puerto serie a un PC con internet. Este subsistema, mostrado en la figura 5, actúa meramente de intermediario entre el subsistema web y el subsistema hoja. Las funciones principales a realizar por dicho subsistema son las siguientes: 
- Cada vez que un dispositivo hoja se conecta manda un mensaje de bienvenida. El subsistema coordinador recibe un evento del tipo "tcpip_event" y mediante el manejador tcpip diseñado, guarda su dirección ip, para tener un control total de los dispositivos hoja que tiene a su alcance.

- Por otra parte este subsistema está escuchando por la UART los mensajes enviados por los usuarios desde el subsistema web. Si un usuario ha decidido realizar alguna acción sobre la farola, enviará el mensaje (acción, ip), y en tal caso, el subsistema coordinador reenviara el mensaje (acción) a dicha farola llamando a la función send_packet.

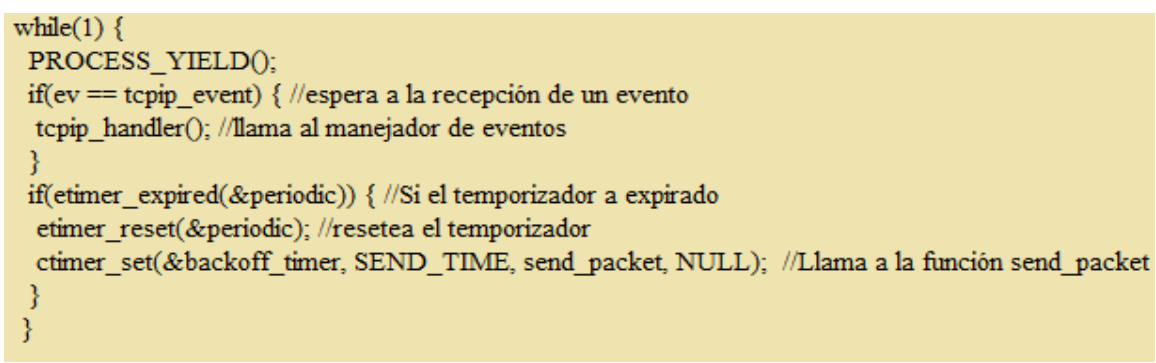

Figura 5. Implementación subsistema coordinador.

\subsection{Subsistema hoja}

Por último, el circuito realizado para el nodo hoja del sistema de alumbrado inteligente se puede ver en la figura 6, donde se aprecia la placa de led encargada de proporcionar luz eficiente, el sensor de movimiento encargado de avisar al sistema en el caso de detectar transeúntes y el detector de luminosidad encargado de proporcionar al sistema la cantidad de luz ambiental.

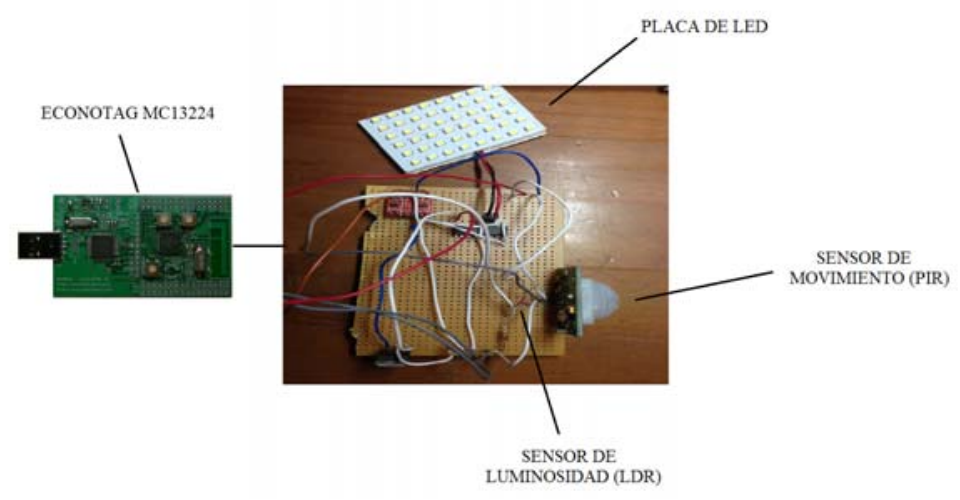

Figura 6. Nodo hoja del sistema de alumbrado inteligente. 
Este subsistema es el encargado de interaccionar directamente con la farola (ajustándole su intensidad) en función de los datos proporcionados por sus sensores.

Como podemos observar en la figura 7, el subsistema hoja inicializa los parámetros necesarios para poder utilizar los sensores y recoge los resultados cada cierto tiempo. En el caso de que el sensor de movimiento dé una respuesta afirmativa la luz se encenderá, y si es el sensor de luminosidad el que avisa de que la cantidad lumínica ha variado se ajustará la intensidad lumínica de la farola en función del valor proporcionado. Por otro lado este subsistema escuchará cada cierto tiempo los mensajes recibidos vía 6lowpan y en el caso de que el usuario haya indicado alguna acción, realizará las acciones pertinentes.

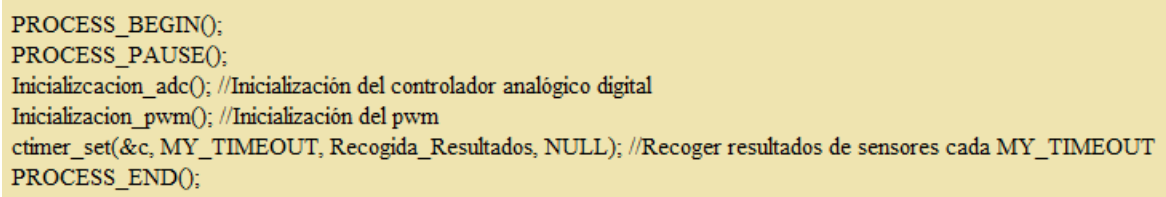

Figura 7. Implementación subsistema hoja.

\subsubsection{Modulación por ancho de pulso (PWM)}

Una de las tareas más importantes de este trabajo ha sido conseguir diferentes intensidades de luz ya que un led solo tiene dos funciones: cuando se supera un determinado voltaje el led se enciende, mientras que si el voltaje baja de ese umbral el led permanece apagado. Para el funcionamiento de la plataforma de alumbrado inteligente, era estrictamente necesario conseguir regular el nivel de intensidad y se ha conseguido gracias a la dimerización de los leds mediante la modulación por ancho de pulsos (PWM) a nivel de software ya que el microcontrolador MC133324 de Freescale no tenía esta característica implementada por hardware.

La modulación por ancho de pulso (PWM) (pulse width modulation) de una señal o fuente de energía es una técnica en la que se modifica el ciclo de trabajo (D) de una señal periódica (una sinusoidal o cuadrada), ya sea para transmitir información a través de un canal de comunicaciones o para controlar la cantidad de energía que se envía a una carga (ver figura 8). 

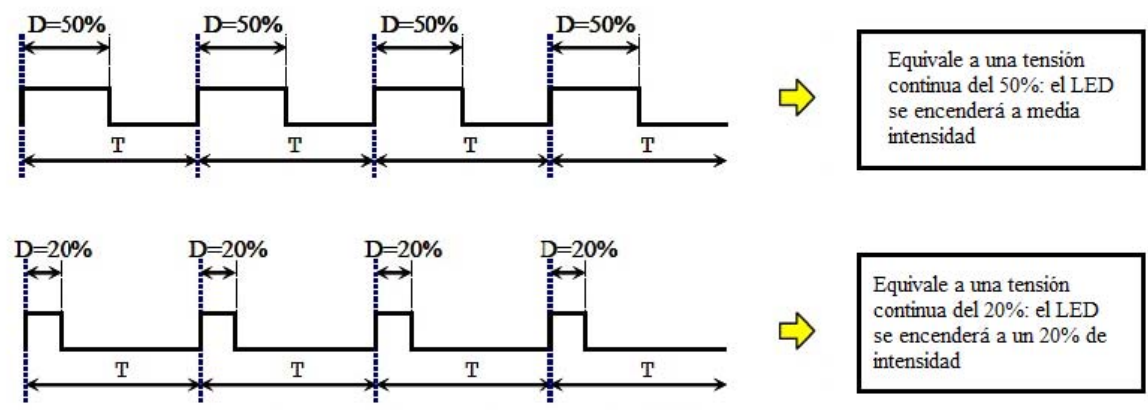

Figura 8. Modulación por ancho de pulso PWM.

El ciclo de trabajo de una señal periódica es el ancho relativo de su parte positiva en relación con el período. Expresado matemáticamente:

D : es el ciclo de trabajo.

$\tau$ : es el tiempo en que la función es positiva (ancho del pulso).

$\mathrm{T}$ : es el período de la función.

$$
D=\frac{\tau}{T}
$$

En concreto, en el subsistema hoja se ha definido un PWM con 5 niveles de luz, de tal manera que un LED pueda regular su intensidad en los siguientes rangos: $0 \%$. $25 \%$, $50 \%, 75 \%, 100 \%$. De esta forma, dependiendo del nivel de luminosidad proporcionado por el sensor LDR, se decidirá qué porcentaje es el más adecuado y se generará el tren de impulsos correspondiente.

\section{Resultados}

En este apartado se van a exponer todas las pruebas realizadas para comprobar el correcto funcionamiento de los diferentes módulos que componen el sistema. Se van a proponer diferentes test sobre los posibles casos de uso más importantes que se pueden dar en el sistema. 
En esta primera prueba se va a intentar simular el paso de una persona. Según se ha diseñado, la farola puede encenderse o no encenderse, dependiendo de los siguientes casos:

- Si hay mucha luz y se detecta movimiento -> no hace falta encender la farola para que vean las personas, ya que con la luz ambiental disponible es suficiente.

- Si hay poca luz y se detecta movimiento -> se enciende la farola para permitir a los transeúntes pasear con total normalidad.

Podemos comprobar en la figura 9 que en estas dos situaciones sucede lo correcto. En la foto de la izquierda la luz ambiental es relativamente baja, por lo que es conveniente encender la farola en caso de movimiento. Por otro lado, en la foto de la derecha, hay gran cantidad de luz, y aunque el detector de movimiento informe de que hay personas andando, no es necesario encender la farola.

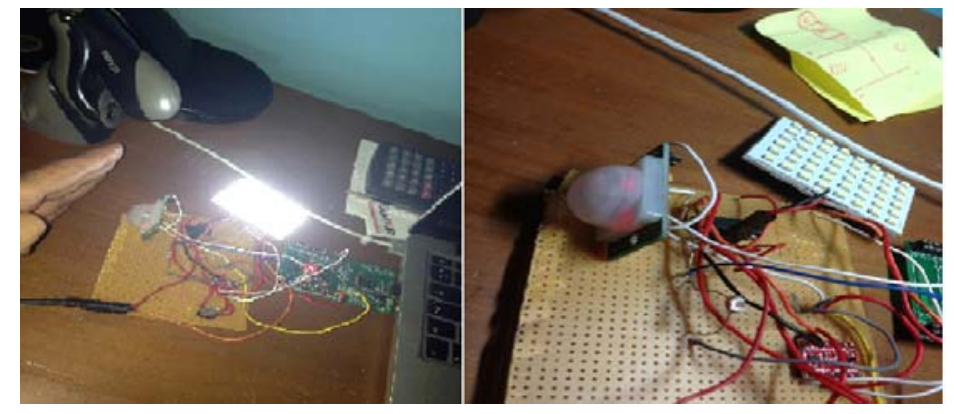

Figura 9. Prueba detección de movimiento.

En esta segunda prueba se va a simular el detector de luminosidad instalado en el sistema. Según se ha diseñado, la farola debe encenderse a una determinada intensidad dependiendo de la cantidad de luz ambiental que se tenga.

- Si hay mucha luz -> No hace falta encender la farola.

- Si hay luz media -> Es necesario encender la farola a un 50\% de su intensidad total para así conseguir el óptimo de luz ambiental.

- Si hay poca luz -> En este caso, la farola se enciende al $100 \%$ de su intensidad ya que se necesita contrarrestar la baja cantidad de luz ambiental.

Podemos comprobar en la figura 10 que en estas tres situaciones sucede lo correcto. En la foto de la izquierda se encuentra el caso de uso donde la luz ambiental es muy baja y la farola se enciende al $100 \%$, en la foto central la farola se enciende al $50 \%$ de su intensidad ya que existe una luz ambiental media, y por último en la foto de la derecha la farola no se enciende porque la cantidad de luz ambiental es muy alta. 


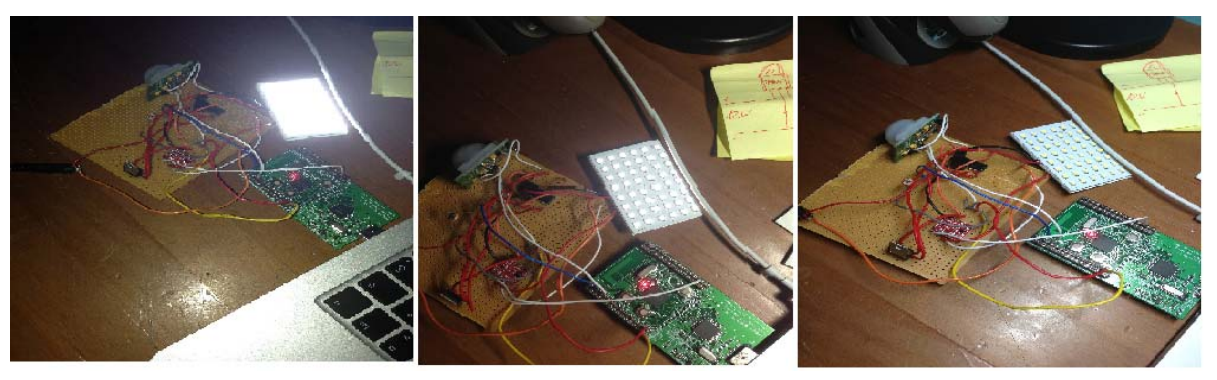

Figura 10. Prueba detección de luminosidad.

Una vez terminado este sistema se puede calcular cuánto se podría llegar a ahorrar en un caso práctico. Supongamos que tenemos una vía muy poco transitada a la entrada de una población en la que hay instaladas 50 luminarias LED inteligentes de 50 vatios de potencia, que han sustituido a los anteriores modelos de 150 vatios de sodio. Si no anda nadie en los alrededores su consumo baja en un 70\%, hasta situarse en unos 15 vatios de potencia que aportan una uniformidad y luz de fondo más que suficiente. $\mathrm{Si}$ se detecta presencia suben a un $100 \%$ pasando a consumir unos 50 vatios. Si en el trayecto no hay presencia durante el $80 \%$ del tiempo, el ahorro energético obtenido es superior al $85 \%$, ya que se pasa de tener un gasto de unos 54000 vatios al año a 8030 vatios, lo que supone un gran ahorro si se instalara en grandes ciudades como Madrid o Barcelona.

Una vez visto el resultado de las pruebas experimentales, el proyecto fin de carrera puede parecer bastante básico, pero detrás de todos estos ensayos hay una gran labor de investigación y aprendizaje para el estudiante que acaba de terminar su formación académica en una titulación en la que no se han tratado los temas con los que ha tenido que lidiar, y solamente la asignatura de Redes de Computadores ha ayudado en temas de comunicación.

\section{Conclusiones}

El objetivo de este documento es la presentación del trabajo fin de carrera "Iluminación inteligente en las ciudades del futuro" en la titulación de Ingeniería Informática de la Universidad de Granada.

El trabajo defendido en Septiembre de 2013 presenta un sistema de alumbrado público inteligente, con alto grado de adaptabilidad y facilidad de instalación. El sistema se aprovecha de una estrategia de control centralizada y el uso de elementos de conexión inalámbrica para lograr una mayor eficiencia energética. El sistema propuesto puede acomodarse a las diferentes necesidades de cada municipio asegurando la escalabilidad, la interoperabilidad y la accesibilidad (en el sentido de que el sistema es accesible desde múltiples plataformas: teléfono móvil, PC, Tablet). 
Bajo la opinión del primer autor, el plan de estudios de las antiguas titulaciones estaba muy desfasado y lejos de lo que realmente se está viviendo actualmente en el mundo de la Informática. Sin embargo, con la adopción del nuevo plan de estudios del Grado de Ingeniería Informática se han corregido la mayoría de estas carencias ofreciendo al alumnado asignaturas mucho más cercanas a lo demandado en al ámbito profesional.

\section{Referencias}

1. World Urbanization Prospects, United Nations, Dept. of Economic and Social Affairs, Population Division, 2011 revision, 2012.

2. Instituto para la Diversificación y Ahorro de la Energía (IDAE). Disponible online: http://www.idae.es/index.php/id.644/relmenu.355/lang.uk/mod.pags/mem.detalle (accedido el 12 Marzo 2014).

3. Lighting of Streets and Buildings in Cities. Disponible online: http://energythic.com/view.php?node=406 (accedido el 12 Marzo 2014).

4. G. Piro, I. Cianci, L.A. Grieco, G. Boggia, P. Camarda. Information centric services in Smart Cities, JOURNAL OF SYSTEMS AND SOFTWARE Volumen: 88 Páginas: 169188 Fecha de publicación: FEB 2014.

5. Miguel Castro, Antonio J. Jara and Antonio F. G. Skarmeta. Smart Lighting solutions for Smart Cities, IEEE 27TH INTERNATIONAL CONFERENCE ON ADVANCED INFORMATION NETWORKING AND APPLICATIONS WORKSHOPS (WAINA) Páginas: 1374-1379 Fecha de publicación: 2013.

6. Reference Manual MC1322x, Document number: 1322xRM; Rev.0.0; 08/2008.

7. Dunkels, A; Grönvall, Björn; Voigt, Thiemo. Contiki- a Lightweight and Flexible operating System for Tiny Networked Sensors. Swedish Institute of Computer Science, PROCEEDINGS - CONFERENCE ON LOCAL COMPUTER NETWORKS Páginas: 455-462 Fecha de publicación: 2004.

8. Guido Moritz, Frank Golatowski, Christian Lerche, and Dirk Timmermann. 6LoWPAN: a study on QoS security threats and countermeasures using intrusion detection system approach, NTERNATIONAL JOURNAL OF COMMUNICATION SYSTEMS Volumen: 25 Número: 9 Número especial: SI Páginas: 1189-1212 Fecha de publicación: SEP 2012. 